\title{
Numerical Solution of Unsteady Radiative Flow Past an Oscillating Semi-Infinite Vertical Plate with Uniform Mass Flux
}

\author{
R. Muthucumaraswamy*, B. Saravanan \\ Department of Applied Mathematics, Sri Venkateswara College of Engineering \\ Pennalur, Sriperumbudur 602105, India \\ *e-mail:msamy@svce.ac.in
}

Received: 20 March 2012; revised: 15 November 2012; accepted: 15 November 2012; published online: 27 February 2013

\begin{abstract}
Thermal radiation effects on unsteady flow past an oscillating semi-infinite isothermal vertical plate with uniform mass flux have been studied. The fluid considered here is a gray, absorbing-emitting radiation but non-scattering medium. The dimensionless governing equations are solved by an efficient, more accurate, and unconditionally stable and fast converging implicit scheme. The effect of velocity and temperature for different parameters like thermal radiation, Schmidt number, thermal Grashof number and mass Grashof number are studied. It is observed that the velocity decreases in the presence of thermal radiation.
\end{abstract}

Key words: radiation, isothermal, vertical plate, finite-difference, mass flux

\section{INTRODUCTION}

Radiative heat and mass transfer play an important role in manufacturing industries for the design of reliable equipment. Nuclear power plants, gas turbines and various propulsion devices for aircraft, missiles, satellites and space vehicles are examples of such engineering applications. Radiative convective flows are encountered in countless industrial and environment processes e.g. heating and cooling chambers, fossil fuel combustion energy processes, evaporation from large open water reservoirs, astrophysical flows, solar power technology and space vehicle re-entry.

England and Emery [1] have studied the thermal radiation effects of a optically thin gray gas bounded by a stationary vertical plate. Soundalgekar and Takhar [2] have considered the radiative free convective flow of optically thin gray-gas past a semi-infinite vertical plate. Radiation effect on mixed convection along an isothermal vertical plate were studied by Hossain and Takhar [3]. In all above-mentioned studies, the stationary vertical plate is considered. Raptis and Perdikis [4] have studied the effects of thermal radiation and free convection flow past a moving infinite vertical plate. Again, Raptis and Perdikis [5] studied thermal radiation effects on a moving infinite vertical plate in the presence of mass diffusion. Radiation effects on a moving infinite vertical plate with variable temperature were studied by Muthucumaraswamy and Ganesan [6]. The dimensionless governing equations were solved by the placeLaplace transform technique.

The flow of a viscous, incompressible fluid past an infinite isothermal vertical plate, oscillating in its own plane, was solved by Soundalgekar [7]. The effect on the flow past a vertical oscillating plate due to a combination of concentration and temperature differences was studied extensively by Soundalgekar and Akolkar [8]. The effect of mass transfer on the flow past an infinite vertical oscillating plate in the presence of constant heat flux has been studied by Soundalgekar et al. [9].

A numerical solution on natural convection along an oscillating isothermal vertical plate under the combined buoyancy effects of heat and mass diffusion in the presence of thermal radiation has not received attention of any researcher. This study was found useful in distribution of cooling in a closed environment. Hence, the present study is to investigate the unsteady flow past an oscillating semi-infinite vertical plate with thermal radiation by an implicit finite-difference scheme of Crank-Nicolson type. 


\section{MATHEMATICAL FORMULATION OF THE PROBLEM}

A transient, laminar, unsteady natural convection flow of a viscous incompressible fluid past an oscillating semiinfinite isothermal vertical plate in the presence of thermal radiation has been considered. It is assumed that the concentration $C^{\prime}$ of the diffusing species in the binary mixture is very little in comparison to the other chemical species which are present. Here, the $x$-axis is taken along the plate in the vertically upward direction and the $y$-axis is taken normally to the plate. The physical model of the problem is shown in Figure 1.
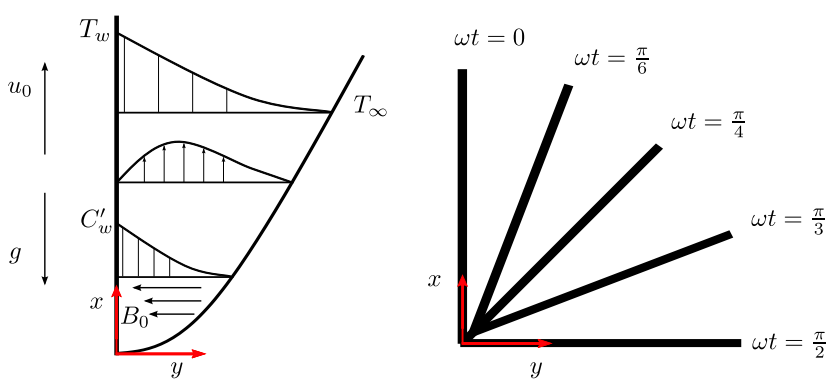

Fig. 1. Physical model of the problem

Initially,it is assumed that the plate and the fluid are of the same temperature and concentration. At time $t^{\prime}>0$, the plate starts oscillating in its own plane with frequency $\omega^{\prime}$ against gravitational field. The temperature of the plate is raised $T_{w}$ and the concentration level near the plate is raised at a constant rate. The fluid considered here is a gray, absorbing-emitting radiation but a non-scattering medium and the viscous dissipation is assumed to be negligible. Then under the above assumptions, the governing boundary layer equations of mass, momentum and concentration for free convective flow with usual Boussinesq's approximation are as follows (Gebart and Pera [10]):

$$
\begin{gathered}
\frac{\partial u}{\partial x}+\frac{\partial v}{\partial y}=0 \\
\frac{\partial u}{\partial t^{\prime}}+u \frac{\partial u}{\partial x}+v \frac{\partial u}{\partial y}= \\
=g \beta\left(T^{\prime}-T_{\infty}\right)+g \beta^{*}\left(C^{\prime}-C_{\infty}^{\prime}\right)+\nu \frac{\partial^{2} u}{\partial y^{2}} \\
\rho C_{p}\left(\frac{\partial T^{\prime}}{\partial t^{\prime}}+u \frac{\partial T^{\prime}}{\partial x}+v \frac{\partial T^{\prime}}{\partial y}\right)=\frac{\partial^{2} T^{\prime}}{\partial y^{2}}-\frac{\partial q_{r}}{\partial y} \\
\frac{\partial C^{\prime}}{\partial t^{\prime}}+u \frac{\partial C^{\prime}}{\partial x}+v \frac{\partial C^{\prime}}{\partial y}=D \frac{\partial^{2} C^{\prime}}{\partial y^{2}} .
\end{gathered}
$$

The initial and boundary conditions are

$$
\begin{aligned}
& t^{\prime} \leq 0 \text { : } \\
& u=0, v=0, T^{\prime}=T_{\infty}, C^{\prime}=C_{\infty}^{\prime} \\
& t^{\prime}>0 \text { : } \\
& \left.\begin{array}{c}
u=u_{0} \cos \omega^{\prime} t^{\prime}, v=0, T^{\prime}=T_{w}, \\
\frac{\partial C^{\prime}}{\partial y}=-\frac{j^{\prime \prime}}{D}
\end{array}\right\} \text { at } y=0, \\
& u=0, T^{\prime}=T_{\infty}, C^{\prime}=C_{\infty}^{\prime}, \quad \text { at } x=0, \\
& u \rightarrow 0, T^{\prime} \rightarrow T_{\infty}, C^{\prime} \rightarrow C_{\infty}^{\prime}, \quad \text { as } y \rightarrow \infty
\end{aligned}
$$

In the case of an optically thin gray gas the local radiant absorption is expressed by

$$
\frac{\partial q_{r}}{\partial y}=-4 a^{*} \sigma\left(T_{\infty}^{4}-T^{\prime 4}\right) .
$$

We assume that the temperature differences within the flow are sufficiently small such that $T^{4}$ may be expressed as a linear function of the temperature. This is accomplished by expanding $T^{4}$ in a Taylor series about $T_{\infty}$ and neglecting higher-order terms, thus

$$
T^{\prime 4} \cong 4 T_{\infty}^{3} T^{\prime}-3 T_{\infty}^{4}
$$

By using equations (6) and (7), equation (3) reduces to

$$
\begin{aligned}
& \rho C_{p}\left(\frac{\partial T^{\prime}}{\partial t^{\prime}}+u \frac{\partial T^{\prime}}{\partial x}+v \frac{\partial T^{\prime}}{\partial y}\right)= \\
= & k \frac{\partial^{2} T^{\prime}}{\partial y^{2}}+16 a^{*} \sigma T_{\infty}^{3}\left(T_{\infty}-T^{\prime}\right) .
\end{aligned}
$$

On introducing the following non-dimensional quantities

$$
\begin{gathered}
U=\frac{u}{u_{0}}, \quad V=\frac{v}{u_{0}}, \quad t=\frac{t^{\prime} u_{0}^{2}}{\nu}, \quad X=\frac{x u_{0}}{\nu}, \\
Y=\frac{y u_{0}}{\nu}, \quad T=\frac{T^{\prime}-T_{\infty}}{T_{w}-T_{\infty}} \\
G r=\frac{g \beta \nu\left(T_{w}-T_{\infty}\right)}{u_{0}^{3}}, \quad C=\frac{C^{\prime}-C_{\infty}^{\prime}}{\left(\frac{j^{\prime \prime} \nu}{D u_{0}}\right)}, \\
G c=\frac{\nu^{2} g \beta^{*} j^{\prime \prime}}{D u_{0}^{4}}, \quad \omega=\frac{\omega^{\prime} \nu}{u_{0}^{2}}, \\
R=\frac{16 a^{*} \nu^{2} \sigma \quad T_{\infty}^{3}}{k u_{0}^{2}}, \quad \operatorname{Pr}=\frac{\mu C_{p}}{k}, \quad S c=\frac{\nu}{D} .
\end{gathered}
$$

Equations (1) to (4) are reduced to the following nondimensional form

$$
\frac{\partial U}{\partial X}+\frac{\partial V}{\partial Y}=0
$$




$$
\begin{gathered}
\frac{\partial U}{\partial t}+U \frac{\partial U}{\partial X}+V \frac{\partial U}{\partial Y}=G r T+G c C+\frac{\partial^{2} U}{\partial Y^{2}} \\
\frac{\partial T}{\partial t}+U \frac{\partial T}{\partial X}+V \frac{\partial T}{\partial Y}=\frac{1}{\operatorname{Pr}} \frac{\partial^{2} T}{\partial Y^{2}}-\frac{R}{P r} T \\
\frac{\partial C}{\partial t}+U \frac{\partial C}{\partial X}+V \frac{\partial C}{\partial Y}=\frac{1}{S c} \frac{\partial^{2} C}{\partial Y^{2}}
\end{gathered}
$$

$$
\begin{aligned}
& \frac{\left[T_{i, j}^{n+1}-T_{i, j}^{n}\right]}{\Delta t}+U_{i, j}^{n} \frac{\left[T_{i, j}^{n+1}-T_{i-1, j}^{n+1}+T_{i, j}^{n}-T_{i-1, j}^{n}\right]}{2 \Delta X} \\
+ & V_{i, j}^{n} \frac{\left[T_{i, j+1}^{n+1}-T_{i, j-1}^{n+1}+T_{i, j+1}^{n}-T_{i, j-1}^{n}\right]}{4 \Delta Y}= \\
= & \frac{1}{\operatorname{Pr}} \frac{\left[T_{i, j-1}^{n+1}-2 T_{i, j}^{n+1}+T_{i, j+1}^{n+1}+T_{i, j-1}^{n}-2 T_{i, j}^{n}+T_{i, j+1}^{n}\right]}{2(\Delta Y)^{2}} \\
- & \frac{R\left(T_{i, j}^{n+1}+T_{i, j}^{n}\right)}{2 P r},
\end{aligned}
$$

The corresponding initial and boundary conditions in nondimensional quantities are

$$
\begin{aligned}
& t \leq 0 \text { : } \\
& U=0, V=0, T=0, C=0, \\
& t>0 \text { : } \\
& \left.\begin{array}{rl}
U= & \cos \omega t, V=0, T=1, \\
& \frac{\partial C}{\partial Y}=-1
\end{array}\right\} \quad \text { at } Y=0, \\
& U=0, T=0, C=0, \quad \text { at } X=0, \\
& U \rightarrow 0, T \rightarrow 0, C \rightarrow 0, \quad \text { as } Y \rightarrow \infty .
\end{aligned}
$$

\section{NUMERICAL TECHNIQUE}

In order to solve the unsteady, non-linear coupled equations (10) to (13) under the conditions (14), an implicit finite difference scheme of Crank- Nicolson type has been employed. The finite difference equations corresponding to equations (10) to (13) are as follows

$$
\begin{gathered}
\frac{\left[U_{i, j}^{n+1}-U_{i-1, j}^{n+1}+U_{i, j}^{n}-U_{i-1, j}^{n}\right]}{4 \Delta X} \\
+\frac{\left[U_{i, j-1}^{n+1}-U_{i-1, j-1}^{n+1}+U_{i, j-1}^{n}-U_{i-1, j-1}^{n}\right]}{4 \Delta X} \\
+\frac{\left[V_{i, j}^{n+1}-V_{i, j-1}^{n+1}+V_{i, j}^{n}-V_{i, j-1}^{n}\right]}{2 \Delta Y}=0, \\
\frac{\left[U_{i, j}^{n+1}-U_{i, j}^{n}\right]}{\Delta t}+U_{i, j}^{n} \frac{\left[U_{i, j}^{n+1}-U_{i-1, j}^{n+1}+U_{i, j}^{n}-U_{i-1, j}^{n}\right]}{2 \Delta X} \\
+V_{i, j}^{n} \frac{\left[U_{i, j+1}^{n+1}-U_{i, j-1}^{n+1}+U_{i, j+1}^{n}-U_{i, j-1}^{n}\right]}{4 \Delta Y}= \\
=\frac{G r}{2}\left[T_{i, j}^{n+1}+T_{i, j}^{n}\right]+\frac{G c}{2}\left[C_{i, j}^{n+1}+C_{i, j}^{n}\right] \\
+\frac{\left[U_{i, j-1}^{n+1}-2 U_{i, j}^{n+1}+U_{i, j+1}^{n+1}+U_{i, j-1}^{n}-2 U_{i, j}^{n}+U_{i, j+1}^{n}\right]}{2(\Delta Y)^{2}},
\end{gathered}
$$

$$
\begin{aligned}
& \frac{\left[C_{i, j}^{n+1}-C_{i, j}^{n}\right]}{\Delta t}+U_{i, j}^{n} \frac{\left[C_{i, j}^{n+1}-C_{i-1, j}^{n+1}+C_{i, j}^{n}-C_{i-1, j}^{n}\right]}{2 \Delta X} \\
+ & V_{i, j}^{n} \frac{\left[C_{i, j+1}^{n+1}-C_{i, j-1}^{n+1}+C_{i, j+1}^{n}-C_{i, j-1}^{n}\right]}{4 \Delta Y}= \\
= & \frac{1}{S c} \frac{\left[C_{i, j-1}^{n+1}-2 C_{i, j}^{n+1}+C_{i, j+1}^{n+1}+C_{i, j-1}^{n}-2 C_{i, j}^{n}+C_{i, j+1}^{n}\right]}{2(\Delta Y)^{2}} .
\end{aligned}
$$

The concentration boundary condition at $Y=0$ in the finite difference form is

$$
\frac{1}{2} \frac{\left[C_{i, 1}^{n+1}+C_{i, 1}^{n}-C_{i,-1}^{n+1}-C_{i,-1}^{n}\right]}{2 \Delta Y}=-1 .
$$

At $Y=0$ (i.e., $j=0$ ) equation (18), becomes

$$
\begin{aligned}
& \frac{\left[C_{i, 0}^{n+1}-C_{i, 0}^{n}\right]}{\Delta t} \\
+ & U_{i, 0}^{n} \frac{\left[C_{i, 0}^{n+1}-C_{i-1,0}^{n+1}+C_{i, 0}^{n}-C_{i-1,0}^{n}\right]}{2 \Delta X}= \\
= & \frac{1}{S c} \frac{\left[C_{i,-1}^{n+1}-2 C_{i, 0}^{n+1}+C_{i, 1}^{n+1}+C_{i,-1}^{n}-2 C_{i, 0}^{n}+C_{i, 1}^{n}\right]}{2(\Delta Y)^{2}} .
\end{aligned}
$$

After eliminating $C_{i,-1}^{n+1}+C_{i,-1}^{n}$ using equation (19), equation (20) reduces to the form

$$
\begin{aligned}
& \frac{\left[C_{i, 0}^{n+1}-C_{i, 0}^{n}\right]}{\Delta t} \\
+ & U_{i, 0}^{n} \frac{\left[C_{i, 0}^{n+1}-C_{i-1,0}^{n+1}+C_{i, 0}^{n}-C_{i-1,0}^{n}\right]}{2 \Delta X}= \\
= & \frac{1}{S c} \frac{\left[C_{i, 1}^{n+1}-C_{i, 0}^{n+1}+C_{i, 1}^{n}-C_{i, 0}^{n}+2 \Delta Y\right]}{(\Delta Y)^{2}} .
\end{aligned}
$$

Here the region of integration is considered as a rectangle with sides $X_{\max }(=1)$ and $Y_{\max }(=20)$, where $Y_{\max }$ corresponds to $Y=\infty$ which lies very well outside both the momentum and energy boundary layers. The maximum of $Y$ was chosen as 20 after some preliminary investigations so that the last two of the boundary conditions (14) are satisfied with in the tolerance limit $10^{-5}$. After experimenting with a few sets of mesh sizes have been fixed at the level 
case, the spatial mesh sizes are reduced by $50 \%$ in one direction, and later in both directions, and the results are compared. It is observed that, when the mesh size is reduced by $50 \%$ in the $Y$-direction, the results differ in the fifth decimal place while the mesh sizes are reduced by $50 \%$ in $X$-direction or in both directions; the results are comparable to three decimal places. Hence, the above mesh sizes have been considered as appropriate for calculation. The coefficients $U_{i, j}^{n}$ and $V_{i, j}^{n}$ appearing in the finite difference equation are treated as constants at any one time step. Here $i$-designates the grid point along the $X$-direction, $j$ along the $Y$-direction and $k$ to the $t$-time. The values of $U, V$ and $T$ are known at all grid points at $t=0$ from the initial conditions.

The computations of $U, V, T$ and $C$ at time level $(n+1)$ using the values at previous time level $(n)$ are carried out as follows: The finite-difference equations (18) and (21) at every internal nodal point on a particular $i$-level constitute a tridiagonal system of equations. The system of equations is solved by using Thomas algorithm as discusses in Carnahan et al. [11]. Thus, the values of $C$ are found at every nodal point for a particular $i$ at $(n+1)^{t h}$ time level. Similarly, the values of $T$ are calculated from equation (17). Using the values of $C$ and $T$ at $(n+1)^{t h}$ time level in the equation (16), the values of $U$ at $(n+1)^{t h}$ time level are found in a similar manner. Thus, the values of $C, T$ and $U$ are known on a particular $i$-level. Finally, the values of $V$ are calculated explicitly using the equation (15) at every nodal point at particular $i$-level at $(\mathrm{n}+1)^{t h}$ time level. This process is repeated for various $i$-levels. Thus the values of $C, T, U$ and $V$ are known, at all grid points in the rectangle region at $(n+1)^{t h}$ time level.

In a similar manner computations are carried out by moving along i-direction. After computing values corresponding to each $i$ at a time level, the values at the next time level are determined in a similar manner. Computations are repeated until the steady-state is reached. The steady-state solution is assumed to have been reached, when the absolute difference between the values of $U$, and as well as temperature $T$ and concentration $C$ at two consecutive time steps are less than $10^{-5}$ at all grid points.

\section{STABILITY ANALYSIS}

The stability criterion of the finite difference scheme for constant mesh sizes are examined using Von-Neumann technique as explained by Carnahan et al.[10]. The general term of the Fourier expansion for $U, T$ and $C$ at a time arbitrarily called $t=0$, are assumed to be of the form $\exp (i \alpha X) \exp (i \beta Y)$ (here $i=\sqrt{-1})$. At a later time $t$, these terms will become,

$$
\begin{aligned}
& U=F(t) \exp (i \alpha X) \exp (i \beta Y), \\
& T=G(t) \exp (i \alpha X) \exp (i \beta Y), \\
& C=H(t) \exp (i \alpha X) \exp (i \beta Y) .
\end{aligned}
$$

Substituting (22) in Equations (16) to (18); under the assumption that the coefficients $U, T$ and $C$ are constants over any one time step and denoting the values after one time step by $F^{\prime}, G^{\prime}$ and $H^{\prime}$. After simplification, we get

$$
\begin{aligned}
& \frac{\left(F^{\prime}-F\right)}{\Delta t}+\frac{U}{2} \frac{\left(F^{\prime}+F\right)(1-\exp (-i \alpha \Delta X))}{\Delta X} \\
+ & \frac{V}{2} \frac{\left(F^{\prime}+F\right) i \sin \beta \Delta Y}{\Delta Y}= \\
= & \frac{\left(G^{\prime}+G\right) G r+\left(H^{\prime}+H\right) G c-M\left(F^{\prime}+F\right)}{2} \\
+ & \frac{\left(F^{\prime}+F\right)(\cos \beta \Delta Y-1)}{(\Delta Y)^{2}}, \\
& \frac{\left(G^{\prime}-G\right)}{\Delta t}+\frac{U}{2} \frac{\left(G^{\prime}+G\right)(1-\exp (-i \alpha \Delta X))}{\Delta X} \\
+ & \frac{V}{2} \frac{\left(G^{\prime}+G\right) \beta \sin \beta \Delta Y}{\Delta Y}= \\
= & \frac{1}{P r} \frac{\left(G^{\prime}+G\right)(\cos \beta \Delta Y-1)}{(\Delta Y)^{2}}-\frac{R}{2 P r}\left(G^{\prime}+G\right), \\
& \frac{\left(H^{\prime}-H\right)}{\Delta t}+\frac{U}{2} \frac{\left(H^{\prime}+H\right)(1-\exp (-i \alpha \Delta X))}{\Delta X} \\
+ & \frac{V}{2} \frac{\left(H^{\prime}+H\right) i \sin \beta \Delta Y}{\Delta Y}= \\
= & \frac{1}{S c} \frac{\left(H^{\prime}+H\right)(\cos \beta \Delta Y-1)}{(\Delta Y)^{2}} .
\end{aligned}
$$

Equations (23) to (25) can be rewritten as,

$$
\begin{aligned}
(1+A) F^{\prime} & =(1-A) F+\frac{G r}{2}\left(G^{\prime}+G\right) \Delta t \\
& +\frac{G c}{2}\left(H^{\prime}+H\right) \Delta t \\
& (1+B) G^{\prime}=(1-B) G \\
& (1+E) H^{\prime}=(1-E) H
\end{aligned}
$$

where,

$$
\begin{aligned}
A & =\frac{U}{2} \frac{\Delta t}{\Delta X}(1-\exp (-i \alpha \Delta X))+\frac{V}{2} \frac{\Delta t}{\Delta Y} i \sin \beta \Delta Y \\
& -(\cos \beta \Delta Y-1) \frac{\Delta t}{(\Delta Y)^{2}}, \\
B & =\frac{U}{2} \frac{\Delta t}{\Delta X}(1-\exp (-i \alpha \Delta X))+\frac{V}{2} \frac{\Delta t}{\Delta Y} i \sin \beta \Delta Y \\
& -\frac{(\cos \beta \Delta Y-1)}{P r} \frac{\Delta t}{(\Delta Y)^{2}}+\frac{R \Delta t}{2 P r}, \\
E & =\frac{U}{2} \frac{\Delta t}{\Delta X}(1-\exp (-i \alpha \Delta X))+\frac{V}{2} \frac{\Delta t}{\Delta Y} i \sin \beta \Delta Y \\
& -\frac{(\cos \beta \Delta Y-1)}{S c} \frac{\Delta t}{(\Delta Y)^{2}} .
\end{aligned}
$$

After eliminating $G^{\prime}$ and $H^{\prime}$ in Equation (26) using Equations (27) and (28), the resultant equation is given by, 


$$
(1+A) F^{\prime}=(1-A) F+G \frac{G r \Delta t}{(1+B)}+H \frac{G c \Delta t}{(1+E)} .
$$

Equations (27) to (29) can be written in the matrix form as follows:

$$
\left[\begin{array}{l}
F^{\prime} \\
G^{\prime} \\
H^{\prime}
\end{array}\right]=\left[\begin{array}{ccc}
\frac{1-A}{1+A} & D_{1} & D_{2} \\
0 & \frac{1-B}{1+B} & 0 \\
0 & 0 & \frac{1-E}{1+E}
\end{array}\right]\left[\begin{array}{l}
F \\
G \\
H
\end{array}\right]
$$

where, $D_{1}=\frac{G r \Delta t}{(1+A)(1+B)}$ and $D_{2}=\frac{G c \Delta t}{(1+A)(1+E)}$ Now, for stability of the finite difference scheme, the modulus of each eigen value of the amplification matrix does not exceed unity. Since the matrix equation (30) is triangular, the eigen values are its diagonal elements. The eigen values of the amplification matrix are, $(1-A) /(1+A),(1-B) /(1+B)$ and $(1-E) /(1+E)$. Assuming that, $U$ is everywhere nonnegative and $V$ is everywhere non-positive, we get

$$
\begin{aligned}
A & =2 a \sin ^{2}\left(\frac{\alpha \Delta X}{2}\right)+2 c \sin ^{2}\left(\frac{\beta \Delta Y}{2}\right) \\
& +i(a \sin \alpha \Delta X-b \sin \beta \Delta Y),
\end{aligned}
$$

where, $a=\frac{U}{2} \frac{\Delta t}{\Delta X}, b=\frac{|V|}{2} \frac{\Delta t}{\Delta Y}, c=\frac{\Delta t}{(\Delta Y)^{2}}$.

Since the real part of $\mathrm{A}$ is greater than or equal to zero, $|(1-A) /(1+A)| \leq 1$ always. Similarly, $|(1-B) /(1+B)| \leq$ 1 and $|(1-E) /(1+E)| \leq 1$.

Hence the finite difference scheme is unconditionally stable. The local truncation error is $O\left(\Delta t^{2}+\Delta Y^{2}+\Delta X\right)$ and it tends to zero as $\Delta t, \Delta X$ and $\Delta Y$ tend to zero. Hence the scheme is compatible. Stability and compatibility ensures convergence.

\section{RESULTS AND DISCUSSION}

The numerical values of the velocity, temperature and concentation are computed for different parameters like radiation parameter, Schmidt number, phase angle, thermal Grashof number and mass Grashof number. The purpose of the calculations given here is to assess the effects of the parameters $\omega, t, R, G r, G c$ and $S c$ upon the nature of the flow and transport. The value of Prandtl number Pr is chosen so that they represent air $(\operatorname{Pr}=0.71)$ and the Schmidt number $S c=0.6$ (Water vapour).

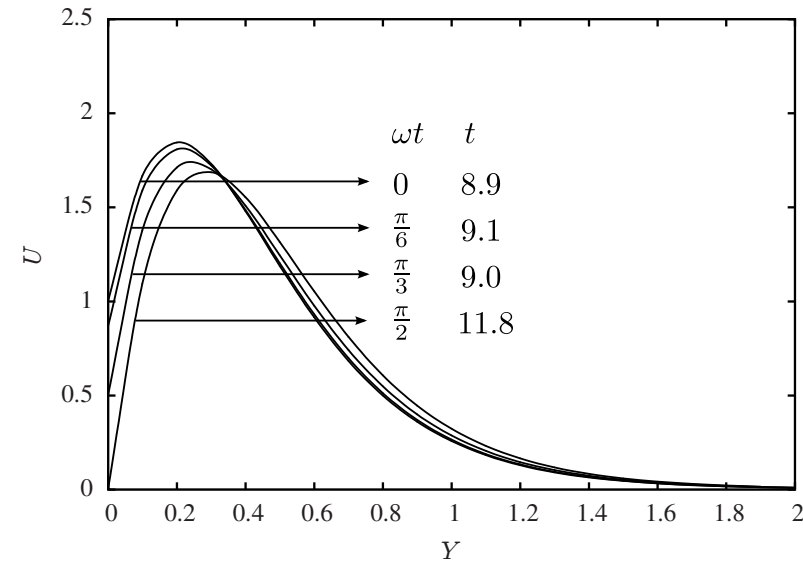

Fig. 2. Steady state velocity profiles for different values of $\omega t$

The steady-state velocity profiles for different phase angle are shown in Figure 2. The velocity profiles presented are those at $X=1.0$. It is observed that for different phase angle ( $\omega t=0, \pi / 6, \pi / 3, \pi / 2), G r=5=G c$ and $R=2$ the velocity decreases with an increasing phase angle. Here $\omega t=0$ represents vertical plate and note that the velocity profile grows from $U=1$ and $\omega t=\pi / 2$ refers horizontal plate and the velocity profiles starting with $U=0$. The numerical value satisfies with the prescribed boundary conditions. It is also observed that the time taken to reach steady-state is more in the case of vertical plate than horizontal plate.

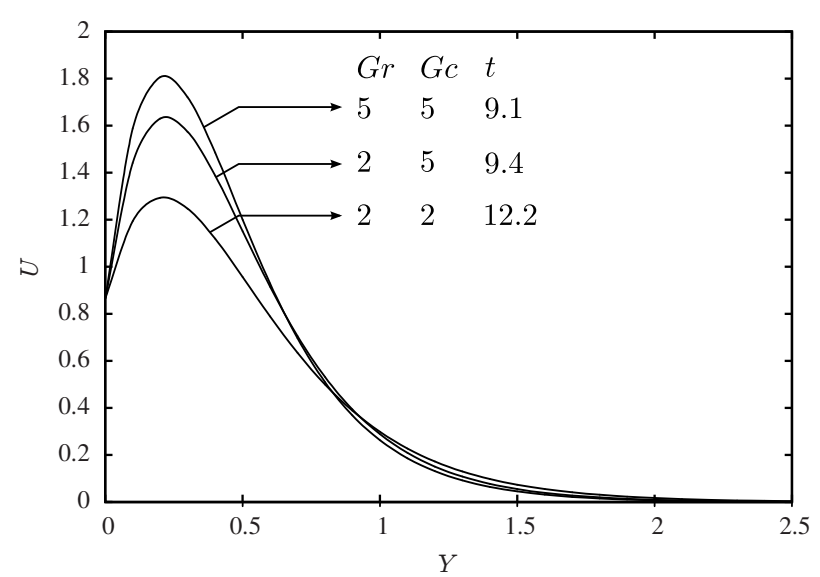

Fig. 3. Steady state velocity profiles for different values of $G r \& G c$

In Figure 3, the velocity profiles for different thermal Grashof number $(G r=2,5)$, mass Grashof number $(G c=2,5), \omega t=\pi / 6$ and $R=2$ are shown graphically. This shows that the velocity increases with increasing thermal Grashof number or mass Grashof number. As thermal Grashof number or mass Grashof number increases, the buoyancy effect becomes more significant, as expected; it implies that, more fluid is entrained from the free stream 
due to the strong buoyancy effects. The effect of velocity for different radiation parameter $(R=0.2,2,5), \omega t=\pi / 6$ and $G r=G c=5$ are shown in Figure 4. It is observed that the velocity increases with decreasing radiation parameter. This shows that velocity decreases in the presence of high thermal radiation.

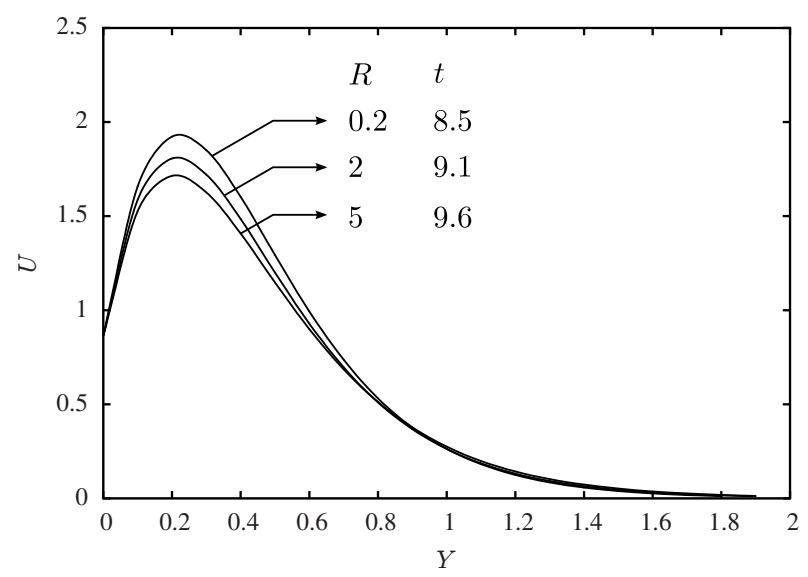

Fig. 4. Velocity profiles for different values of $R$

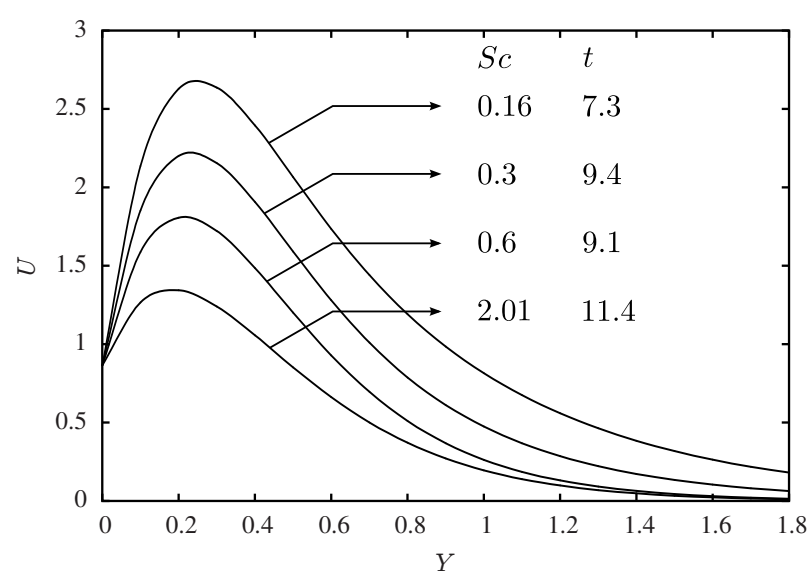

Fig. 5. Velocity profiles for different values of $S c$

The steady-state velocity profiles for a different Schmidt number $(S c=0.16,0.3,0.6,2.01), G r=G c=5, \omega t=\pi / 6$ and $R=2$ are shown in Figure 5 . The presented velocity profiles presented are those at $X=1.0$. It is observed that the velocity decreases with an increasing Schmidt number and the steady-state value increases with an increasing Schmidt number. The velocity boundary layer seems to grow in the direction of motion of the plate. It is observed that near the leading edge of a semi-infinite vertical plate moving in a fluid, the boundary layer develops along the direction of the plate. However, the time required for the velocity to reach steadystate depends upon the Schmidt number. This shows that the contribution of mass diffusion to the buoyancy force increases the maximum velocity significantly. The temperature profiles for different values of the thermal radiation parameter $(R=0.2,2,5,10)$ are shown in Figure 6. It is observed that the temperature increases with decreasing $\mathrm{R}$. This shows that the buoyancy effect on the temperature distribution is very significant in air $(\operatorname{Pr}=0.71)$. It is known that the radiation parameter and Prandtl number plays an important role in flow phenomena because, it is a measure of the relative magnitude of viscous boundary layer thickness to the thermal boundary layer thickness. The concentration profiles for different values of the Schmidt number $(S c=0.16,0.3,0.6,2.01)$ are shown in Figure 7. It is observed that the plate concentration increases with decreasing $S c$.

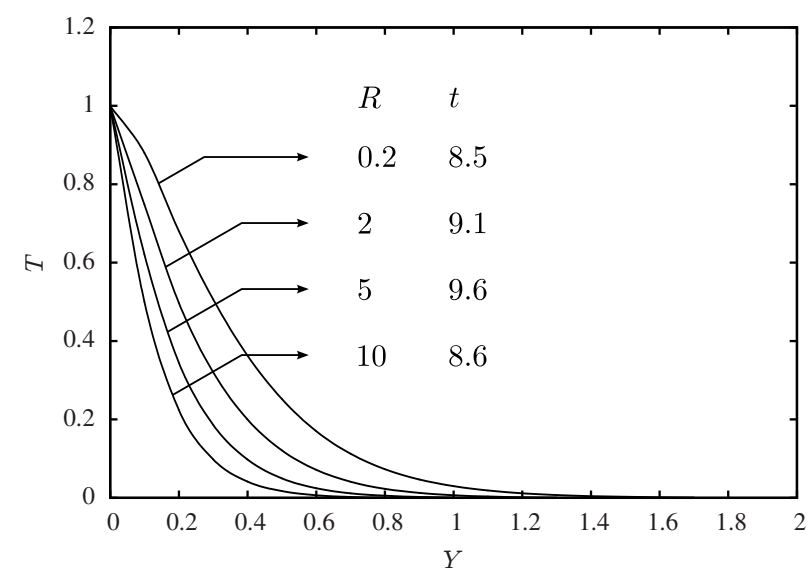

Fig. 6. Temperature profiles for different values of $R$

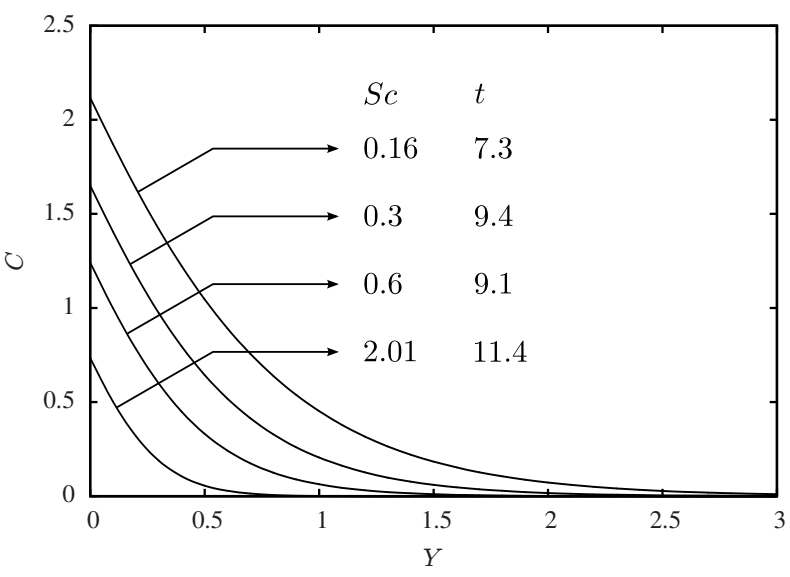

Fig. 7. Concentration profiles for different values of $S c$

Knowing the velocity and temperature field, it is customary to study the skin-friction, Nusselt number and Sherwood number. The local as well as average values of skin-friction, Nusselt number and Sherwood number in dimensionless form are as follows: 


$$
\begin{gathered}
\tau_{X}=-\left(\frac{\partial U}{\partial Y}\right)_{Y=0}, \\
\bar{\tau}=-\int_{0}^{1}\left(\frac{\partial U}{\partial Y}\right)_{Y=0} d X \\
N u_{X}=\frac{-X\left(\frac{\partial T}{\partial Y}\right)_{Y=0}}{T_{Y=0}} \\
\overline{N u}=-\int_{0}^{1}\left[\frac{\left(\frac{\partial T}{\partial Y}\right)_{Y=0}}{T_{Y=0}}\right] d X, \\
S h_{X}=-X\left(\frac{\partial C}{\partial Y}\right)_{Y=0} \\
\overline{S h}=-\int_{0}^{1}\left(\frac{\partial C}{\partial Y}\right)_{Y=0} d X .
\end{gathered}
$$

The derivatives involved in the equations (32) to (37) are evaluated using five-point approximation formula and then the integrals are evaluated using Newton-Cotes closed integration formula.

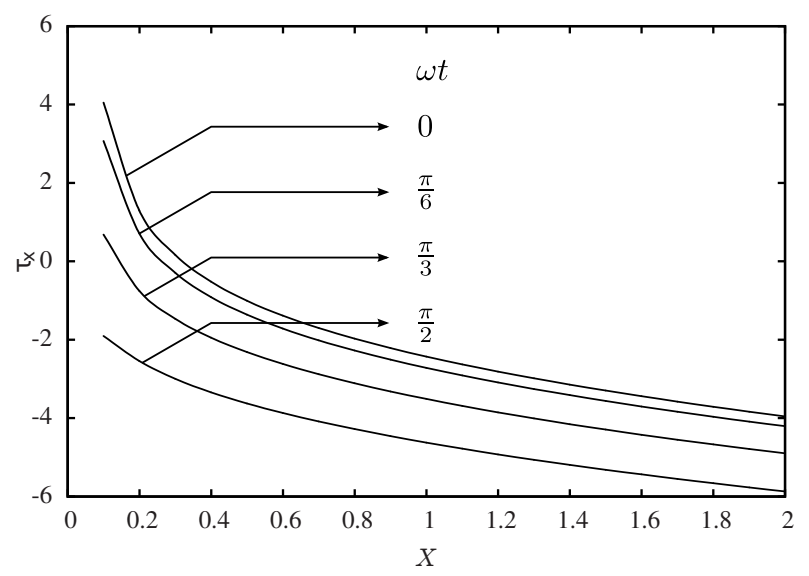

Fig. 8. Local skin friction

The local skin-friction, Nusselt number and Sherwood number are plotted in Figures 8, 9 and 10, respectively. Local skin-friction values for a different phase angle are evaluated from equation (32) and plotted in Figure 8 as a function of the axial coordinate. The local wall shear stress increases with a decreasing phase angle. The trend shows that the wall shear stress is more in the case of vertical plate than horizontal plate. The local Nusselt number for different thermal radiation parameter is presented in Figure 9 as a function of the axial co-ordinate. The trend shows that the Nusselt number increases with increasing values of the thermal radiation parameter. It is clear that the rate of heat transfer is more in the presence of thermal radiation. The local Sherwood number for different values of the Schmidt number are shown in Figure 10. As expected, the rate of mass transfer increases with increasing values of the Schmidt number. This trend is just reversed as compared to the concentration field for a different Schmidt number given in Figure 7.

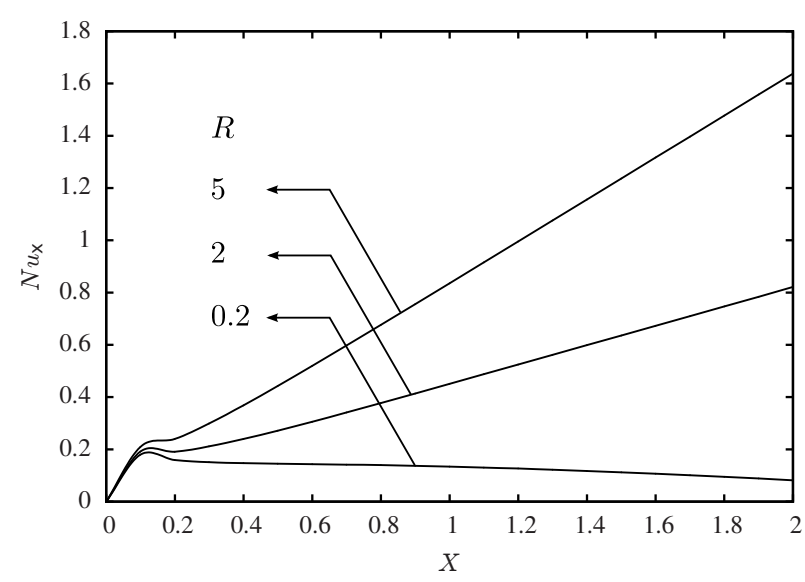

Fig. 9. Local Nusselt number

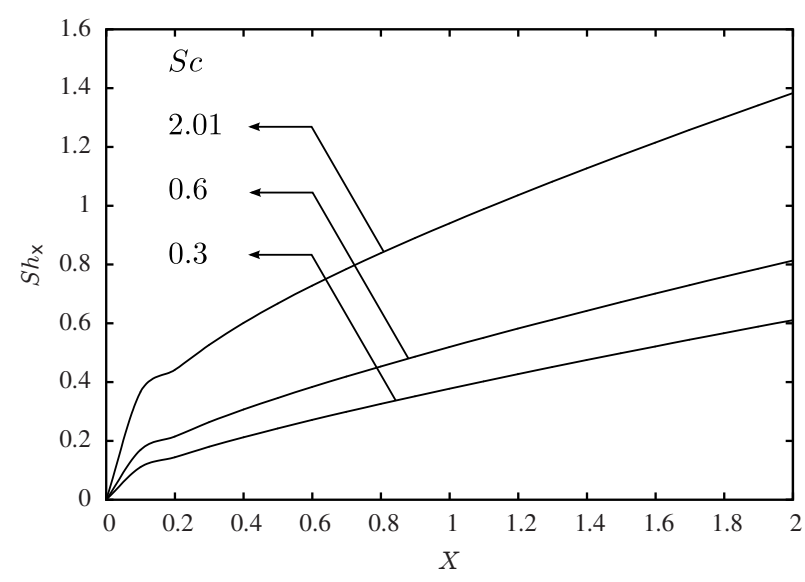

Fig. 10. Local Sherwood number

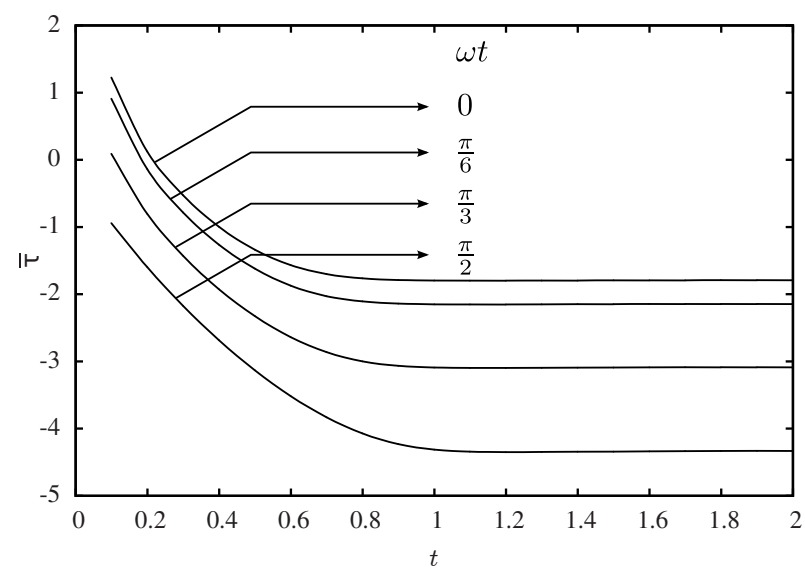

Fig. 11. Average skin friction 


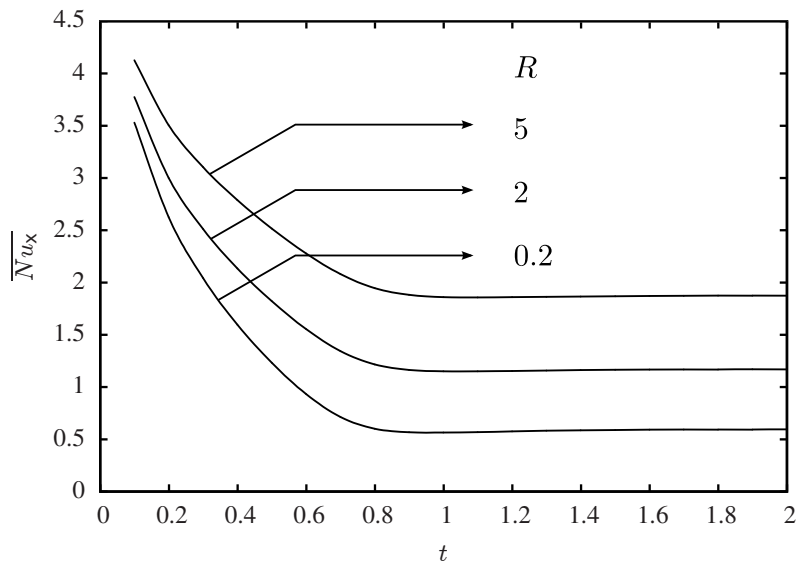

Fig. 12. Average Nusselt number

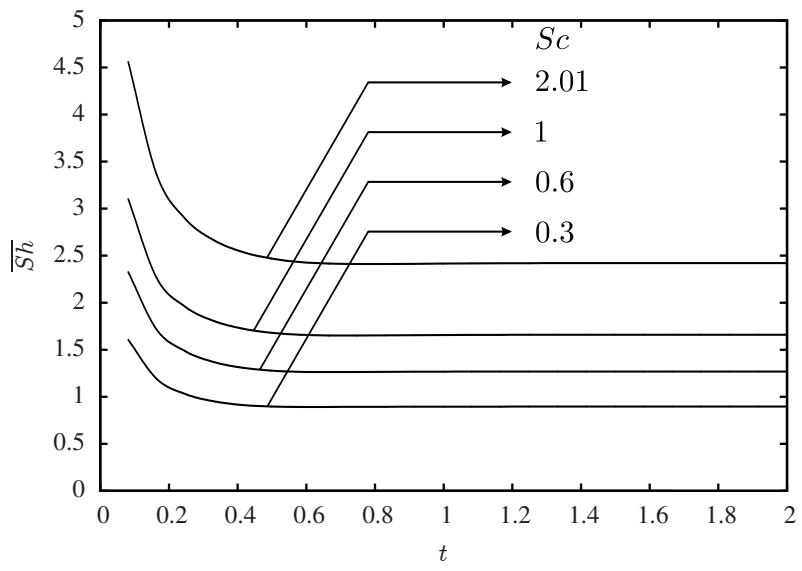

Fig. 13. Average Sherwood number

The average values of the skin-friction, Nusselt number and Sherwood number are shown in Figures 11, 12 and 13, respectively. The effects of the different phase angle on the average values of the skin-friction are shown in Figure 10. The average skin-friction decreases with decreasing with increasing values of the phase angle. Figure 11 illustrates the average Nusselt number increases with increasing radiation parameter. From the Figure 12, it is observed that the average Sherwood number increases with increasing values of the Schmidt number.

\section{CONCLUSIONS}

The numerical study has been carried out for thermal radiation effects on unsteady flow past an oscillating semi-infinite isothermal vertical plate with prescribed uniform mass flux. The dimensionless governing equations are solved by an implicit scheme of Crank-Nicolson type. The effect of velocity, temperature and concentration for different parameter are studied. The local as well as average skin-friction, Nusselt number and Sherwood number are shown graphically. It is observed that the contribution of mass diffusion to the buoyancy force increases the maximum velocity significantly. It is also observed that the velocity decreases in the presence of thermal radiation. The study shows that the number of time steps to reach steady-state depends strongly on the radiation parameter.

\section{Symbols}

$a^{*} \quad$ absorption constants

$C^{\prime} \quad$ species concentration in the fluid

$C$ dimensionless concentration

$C_{w}^{\prime} \quad$ concentration of the plate

$C_{\infty}^{\prime} \quad$ concentration in the fluid far away from the plate

$C_{p} \quad$ specific heat at constant pressure

$D$ mass diffusion coefficient

Gc mass Grashof number

Gr thermal Grashof number

$g \quad$ acceleration due to gravity

$k$ thermal conductivity

$\mathrm{R} \quad$ thermal radiation parameter

$\operatorname{Pr} \quad$ Prandtl number

Sc Schmidt number

M magnetic field parameter

$N u_{x} \quad$ dimensionless local Nusselt number

$\overline{N u}$ dimensionless average Nusselt number

$S h_{x} \quad$ dimensionless local Sherwood number

$\overline{S h}$ dimensionless average Sherwood number

$T$ temperature of the fluid near the plate

$T^{\prime} \quad$ temperature

$T_{w}^{\prime} \quad$ temperature of the plate

$T_{\infty}^{\prime} \quad$ temperature of the fluid far away from the plate

$t^{\prime} \quad$ time

$u_{0} \quad$ velocity of the plate

$u, v \quad$ velocity of the components of the fluid in $\mathrm{X}, \mathrm{Y}$ - directions, respectively

coordinate along the plate

coordinate axis normal to the plate

dimensionless coordinate along the plate

dimensionless coordinate axis normal to the plate thermal difusivity

volumetric coefficient of thermal expansion

volumetric coefficient of expansion with concentration

$\mu \quad$ coefficient of viscosity

$\omega^{\prime} \quad$ frequency of oscillation

$\omega \quad$ dimensional frequency of oscillation

$\omega^{\prime} t^{\prime} \quad$ phase angle

$\omega t$ dimensional phase angle

$\nu \quad$ kinematic viscosity

$\sigma \quad$ electric conductivity 
$\rho \quad$ density of the fluid

$\theta \quad$ dimensionless temperature

$\tau_{x} \quad$ dimensionless local skin friction

$\bar{\tau} \quad$ dimesionless average skin friction

$w \quad$ conditions at the wall

$\infty \quad$ free stream conditions

\section{References}

[1] W.G. England, A.F. Emery, Thermal radiation effects on the laminar free convection boundary layer of an absorbing gas, J. Heat Transfer 91, 37-44 (1969).

[2] V.M.Soundalgekar, H.S.Takhar, Radiation effects on free convection flow past a semi-infinite vertical plate, Journal of Modeling, Measurements and Control B51, 31-40 (1993).

[3] M.A. Hossian, H.S.Takhar, Radiation effect on mixed convection along a vertical plate with uniform surface temperature, Heat and Mass Transfer 31, 243-248 (1996).

[4] A. Raptis, C.Perdikis, Radiation and free convection flow past a moving plate, Int. J. App. Mech. and Eng. 4, 817821(1999).
[5] A. Rpatis, C.Perdikis, Thermal radiation of an optically thin gray gas, Int. J.App. Mech. and Eng. 8, 131-134 (2003).

[6] R.Muthucumaraswamy, P.Ganesan, Radiation effects on flow past an impulsively started infinite vertical plate with variable temperature, Int. J. of Appl. Mech. and Eng. 8, 125-129 (2003).

[7] M.Soundalgekar, Free convection effects on the flow past a vertical oscillating plate, Astrophy. and Space Sci. 64, 165172 (1979).

[8] V.M. Soundalgekar, S.P. Alolkar, Effects of free convection currents and mass transfer on the flow past a vertical oscillating plate, Astrophysics and Space Science 89, 241-254, 1983.

[9] V.M. Soundalgekar, R.M.Lahurikar, S.G.Pohanerkar, N.S.Birajdar, Effects of mass transfer on the flow past an oscillating infinite vertical plate with constant heat flux, Thermophysics and Aero Mech. 1, 119-124 (1994).

[10] B. Gebhart, L. Pera, The nature of vertical natural convection flows resulting from the combined buoyancy effects of thermal and mass diffusion, Int. J. Heat Mass Transfer 14, pp.2025-2050 (1971).

[11] B. Carnahan, H.A. Luther, J.O. Wilkes, Applied Numerical Methods, John Wiley and sons, New York, 1969.

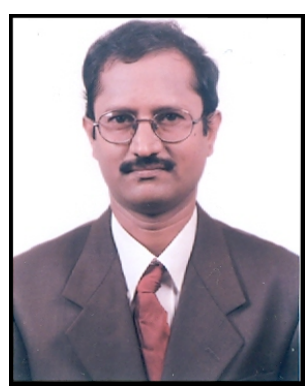

Dr R Muthucumaraswamy, received his B.Sc. degree in Mathematics, from Gurunanank College, University of Madras in 1985, M.Sc. degree in Applied Mathematics from Madras Institute of Technology, Anna University in 1987, M.Phil. degree in Mathematics from Pachaiyappas' College, University of Madras in 1991 and Ph.D. degree in Mathematics from Anna University in 2001. His area of specialization in Theoretical and Computational Fluid Dynamics. He published 180 papers in National/International journals and in conferences. He completed two funded projects from Defence Research and Developmental Organization in 2009 and 2012. He received best teacher award in the year 2001. Currently, working as a Professor and Head, Department of Applied Mathematics, Sri Venkateswara College of Engineering, Sriperumbudur, India.

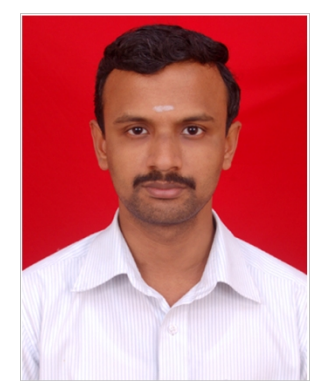

Mr B Saravanan, received his B.Sc. degree in Mathematics from Sacred Heart College, University of Madras in 2000, M.Sc. degree in Mathematics from Ramanujan Institute for Advanced study in Mathematics(RIASM), University of Madras in 2002 and M.Phil. degree in Mathematics, from RIASM, University of Madras in 2003. He received gold medals in B.Sc and M.Sc. Currently working as Assistant Professor, Department of Applied Mathematics, Sri Venkateswara College of Engineering, Sriperumbudur, India. 